by exposure to pathogens or vaccines, which evolved as a protective mechanism against infections. TI is characterized by rewiring of functional, epigenetic and metabolic programs of innate immune cells such as monocytes and macrophages, which sustain enhanced production of pro-inflammatory cytokines. Since aberrant activation of TI is implicated in inflammatory diseases, tight regulatory mechanisms are likely in place, but the mechanisms responsible for this modulation remain elusive.

Objectives Scope of this study was to evaluated the role of IL-37, an anti-inflammatory cytokine that curbs inflammation as well as modulates metabolic pathways, as an endogenous regulator of trained immunity.

Methods The effects of recombinant IL-37 were evaluated in a mouse model of TI induced by the administration of betaglucan in vivo (survival to a lethal inoculum of infectious agents, production of inflammatory cytokines, recruitment of inflammatory cells at the sites of infection). Subsequently, the effects of IL-37 were evaluated ex vivo on splenic and bone marrow monocytes (production of inflammatory cytokines, metabolomic analysis of the activation status of the main pathways of cellular energy metabolism). Finally, we evaluated the association between IL-37 gene polymorphisms and the induction of TI in monocytes of healthy donors with in vitro functional studies.

Results The exogenous administration of IL-37 abrogates the pro-inflammatory effects of TI, significantly reducing the production of pro-inflammatory cytokines and the survival of experimental animals subjected to a disseminated infection model. The inhibitory effects of IL-37 on TI are also associated with reduced recruitment of neutrophils at sites of inflammation. IL-37 and TI programs have differential and opposite effects on the modulation of cellular energy metabolism of monocytes. In humans, polymorphisms in the IL-37 gene are associated with reduced activation of TI programs and reduced production of inflammatory cytokines by healthy donor monocytes.

Conclusions In conclusion, IL-37 emerges as an endogenous regulator of TI, which makes this cytokine a potential therapeutic target in immune-mediated pathologies.

Disclosure of Interest None declared.

\section{P088 CHARACTERIZATION OF NOVEL HUMANIZED IL-17 MOUSE PRECLINICAL PLATFORMS FOR THE EFFICACY EVALUATION OF ANTI-HUMAN IL17 THERAPEUTICS}

${ }^{1} E$ Christodoulou-Vafeiadou*, ${ }^{1} \mathrm{~L}$ Ntari, ${ }^{1} \mathrm{~K}$ Kranidioti, ${ }^{1} \mathrm{MC}$ Denis, ${ }^{1} \mathrm{~N}$ Karagianni, ${ }^{2,3} \mathrm{G}$ Kollias. ${ }^{1}$ BIOMEDCODE Hellas $S A ;{ }^{2}$ Institute of Immunology, BSRC Al. Fleming, Vari; ${ }^{3}$ Department of Physiology, School of Medicine, National Kapodistrian University, Athens, Greece

\subsection{6/annrheumdis-2018-EWRR2019.77}

Career situation of first and presenting author Post-doctoral fellow.

Introduction As the IL17/IL23 axis has proven pathogenic for many inflammatory conditions like rheumatoid arthritis, psoriasis, IBD, multiple sclerosis (MS) and others, IL17 inhibition is an attractive target for the treatment of these diseases. Indeed, biologics targeting human IL17 (hIL17) have recently proven a successful treatment for psoriasis with similar therapeutics being currently under development for this and other diseases.
Objectives To describe novel preclinical platforms based on a hIL17A transgenic mouse model for the efficacy evaluation of human therapeutics, targeting psoriasis and MS.

Methods Human IL17A transgenic mice were generated using as transgene a $170 \mathrm{~kb}$ genomic DNA fragment comprising hIL17A intron exon sequences flanked by extended 5' and 3' regulatory regions. These mice were further crossed with IL17AKO $^{1}$ resulting in a mouse line (TghIL17) that exclusively expresses hIL17A. We standardized IMQ-induced psoriasis and MOG-induced Experimental Autoimmune Encephalomyelitis (EAE) induction protocols in these mice and characterized their response to anti hIL17 treatment. Disease severity was evaluated using established clinical and histopathological scoring scales.

Results TghIL17 mice have no overt pathology, and express hIL17A upon induction. IMQ- induced psoriasis induction in TghIL17 mice resulted in the development of skin pathology characterized clinically by skin erythema, thickening and scaling and histopathologically by acanthosis, hyperkeratosis and lymphocytic infiltration. Treatment with secukinumab resulted in alleviation of both clinical and histopathological psoriasis hallmarks to levels comparable to those observed in the IL17KO mice. MOG-induced EAE in TghIL17 mice resulted in clinical symptoms comparable to the ones observed in WT mice that involved, at the peak of the disease, paraplegia with forelimb weakness or paralysis. Treatment of EAE-affected TghIL17 mice with secukinumab ameliorated the pathological findings to a level similar to the one observed in IL17KO mice.

Conclusions TghIL17 mice upon induction develop pathologies similar to WT animals demonstrating that human IL17A efficiently replaces its mouse counterpart. Using these mice with standardized IMQ-induced psoriasis and MOG-induced EAE protocols we established and validateed with commercially approved anti-hIL17 biologics, preclinical platforms that allow the efficient and reproducible evaluation of anti-hIL17 therapeutics.

\section{REFERENCE}

1. Nakae $S$, et al. Antigen-specific T cell sensitization is impaired in II-17-deficient mice, causing suppression of allergic cellular and humoral responses. Immunity 2002.

Disclosure of Interest None declared.

\section{P089 MUCOSAL-ASSOCIATED INVARIANT T (MAIT)-CELL- DERIVED IL-17A AND IL-17F PRODUCTION IS IL-23- INDEPENDENT AND BIASED TOWARDS IL-17F}

S Cole*, A Maroof. UCB Pharma SA, Slough, UK

10.1136/annrheumdis-2018-EWRR2019.78

Career situation of first and presenting author Young investigator.

Introduction The requirement for IL-23 in driving IL-17A and IL-17F production in humans is incompletely understood. Preclinical data support IL-17F, together with IL-17A, as a key driver of chronic tissue inflammation. ${ }^{1}$ MAIT cells, an innate T-cell population, uniformly express ROR $\gamma$ t but only a minority have been shown to produce IL-17A. ${ }^{2}$ Dysregulation in frequency and function of MAIT cells has been associated with IL-17A-mediated inflammatory diseases, including PsA and AS. $^{3}{ }^{4}$ IL-17F production in MAIT cells remains largely unexplored. 